\title{
Mehr Staat, mehr Markt, mehr Sicherheit Warum die Flüchtlingskrise einen Pfadwechsel in der deutschen Politik der Inneren Sicherheit eingeleitet haben könnte
}

Helge Staff, Georg Wenzelburger

Fachbereich Sozialwissenschaften, TU Kaiserslautern

helge.staff@sowi.uni-kl.de,georg.wenzelburger@sowi.uni-kl.de

\section{Zusammenfassung}

Die Ankunft hunderttausender Flüchtlinge im Herbst 20I5 stellte ungeahnte Herausforderungen an die Akteure im Politikfeld der Inneren Sicherheit in vielen europäischen Staaten. Dieser Artikel fragt daher - bezogen auf den Fall Deutschland welche sicherheitspolitischen Reaktionen die Flüchtlingskrise hervorgerufen hat und inwieweit diese einen Pfadbruch darstellen. Um die Frage zu beantworten, prüfen wir entlang des Konzepts der „critical juncture“ zunächst die Entwicklung verschiedener Indikatoren für Policy-Wandel in der Inneren Sicherheit. In einem zweiten Schritt und auf der Grundlage einer Befragung der deutschen Aufsichtsbehörden zuständig für die Erstaufnahme von Flüchtlingen identifizieren wir dann Mechanismen der Bildung eines neuen Pfades: Ein personell robuster und marktorientierter Policy-Pfad, der ein Zurück in die sicherheitspolitische Zeit vor der Flüchtlingskrise als unwahrscheinlich erscheinen lässt.

\section{Schlüsselwörter}

Flüchtlingskrise, Innere Sicherheit, Private Sicherheit, Pfadabhängigkeit, Deutschland

\section{More State, More Market, More Security Why the refugee crisis might have induced a change of paths in German law \& order policy}

\begin{abstract}
The arrival of hundreds of thousands of refugees in the fall of 2015 posed a major challenge to policymakers in the field of domestic security across Europe. This article thus asks - focusing on the case of Germany - which policy reactions the refugee crisis produced and in how far they amount to a new policy path. Employing the concept of a "critical juncture" we answer this question, first, by looking at a range of indicators of security policy change in Germany. Second, and based on a survey among German agencies responsible for the preliminary accommodation of refugees, we are able to identify mechanisms which could bring about a new path: A policy path characterized by a surge in security personnel and a more market-oriented approach toward law and order, which makes a return to Germany's law and order policy before the refugee crisis very unlikely.
\end{abstract}

\section{Keywords}

Refugee Crisis, Law \& Order, Private Security, Path Dependence, Germany

The authors have declared that no competing interests exist. 


\section{Einleitung ${ }^{1}$}

Das Jahr 20I5 war in vielen europäischen Ländern, ganz besonders aber in Österreich und Deutschland, dadurch gekennzeichnet, dass immer mehr geflüchtete Menschen Zuflucht in den sicheren Staaten Westeuropas suchten. So stieg die Zahl der Asylanträge in Österreich von rund 28.000 im Jahr 2014 auf mehr als $89.000 \mathrm{im}$ Jahr 2015 an (Bundesministerium für Inneres 20I6) - ein ähnlich starker Aufwuchs ist in Deutschland zu beobachten mit einem Anstieg von rund 203.000 im Jahr 2014 auf etwa 477.000 in 2015 bzw. rund 746.000 in 2016 (BAMF 20I7). Die notwendige schnelle Inbetriebnahme von Aufnahmeeinrichtungen war jedoch nicht nur eine humanitäre und integrationspolitische Herausforderung für Bund, Länder und Kommunen in beiden Staaten (Der Standard 20I5; Die Presse 20I4), sondern brachte auch enorme sicherheitspolitische Herausforderungen $^{2}$ mit sich - und zwar in dreifacher Hinsicht: Zum ersten galt es, die innere Ordnung der Flüchtlingsunterkünfte zu gewährleisten, was sich angesichts beengter Verhältnisse und traumatisierter Bewohner unterschiedlicher ethnischer Herkunft und Religion als große Aufgabe erwies. Zum zweiten mussten die Menschen in den Einrichtungen vor Angriffen von außen geschützt werden, die sich nach Angaben des Bundeskriminalamts (BKA) in Deutschland im Verlauf des Jahres mehr als vervierfachten (BKA 20I6; Jäckle/König 20I7). Und drittens ging es auch darum, das Sicherheitsgefühl der Bevölkerung angesichts der außergewöhnlichen sicherheitspolitischen Lage zu adressieren, wie politisierte Diskussionen der Kriminalitätsstatistik oder etwa die jüngsten Wahlen in Österreich zeigen. Angesichts dieser sicherheitspolitischen Herausforderungen der Flüchtlingskrise und unter Heranziehung des Konzepts der critical juncture (Collier/Collier I99I), mit dem ein Ereignis gemeint ist, in dessen Folge in einem Politikfeld ein neuer Policy-Pfad entstehen kann, lautet die Forschungsfrage dieses Artikels: Welche sicherheitspolitischen Reaktionen hat die Flüchtlingskrise hervorgerufen und inwieweit stellen diese einen Pfadbruch dar?

Zur Klärung der Frage fokussiert dieser Beitrag auf die Bundesrepublik Deutschland und nimmt dabei sowohl die Bundes- als auch die Länderebene, sowie staatliche Sicherheitsakteure und die private Sicherheitsbranche in den Blick. In Deutschland wurde im Kontext der Flüchtlingskrise eine Serie sicherheitspolitischer Entscheidungen getroffen - in Form von Gesetzesentwürfen, durch Abkommen zwischen den Organen, aber

I Wir danken den Herausgeber*innen und Gutachter*innen für ihre kritischen und sehr hilfreichen Kommentare zum Manuskript.

2 Diese Herausforderungen entstanden im Feld der Migrationspolitik führten aber auch zu Policy-Wandel in der Inneren Sicherheit auch wenn grundsätzlich von zwei getrennten Politikfeldern auszugehen ist, die oftmals politisch verknüpft werden (Huysmans 20oo, Boswell 2007). auch in vielen kleinen Entscheidungen im Zusammenhang mit praktischen Problemen der Sicherheitsgewährleistung in den Aufnahmeeinrichtungen selbst. Damit, so das Ergebnis unserer Untersuchung, wurde das Politikfeld der Inneren Sicherheit nachhaltig verändert, nachdem es in den vergangenen Jahren eher durch inkrementellen Wandel gekennzeichnet war (Glaeßner 20IO; Staff/Wenzelburger 20I7; Wenzelburger/Staff 20I6). Einige Indizien sprechen sogar in der Tat dafür, dass ein Pfadbruch stattgefunden und ein neuer PolicyPfad begonnen hat.

Dabei ist die Frage der wachsenden Rolle privater Sicherheitsunternehmen in der Sicherheitsproduktion nicht neu. In Folge des allgemeinen Diskurses über die Privatisierung staatlicher Aufgaben entwickelte sich in den I990er und frühen 200oer Jahren in Deutschland eine Debatte über die rechtlichen Möglichkeiten und vor allen Dingen Grenzen einer weitergehenden Verlagerung staatlicher Sicherheitsaufgaben auf private Anbieter (Kötter 2007, 249-255; siehe z.B. Pitschas 2002; Prätorius 2002). In Fortführung dieser Debatten beschreiben Autoren bereits seit einigen Jahren den Wandel der Sicherheitsproduktion in Deutschland (siehe z.B. Beste 2009; Frevel u. Wendekamm 20I7). Der vorliegende Beitrag geht jedoch über diese bestehenden Arbeiten hinaus, in dem er explizit auf theoretische Mechanismen der Pfadabhängigkeit zurückgreift, um auf der Grundlage neuer und vielfältiger Daten das Argument eines speziellen Effekts der Flüchtlingskrise pointiert darzustellen.

Der weitere Beitrag ist in vier Teile gegliedert. Im nächsten Abschnitt diskutieren wir die Theorie der Pfadabhängigkeit und setzen dabei einen Schwerpunkt auf die Mechanismen, welche pfadabhängige Prozesse ermöglichen bzw. brechen. Darauf aufbauend betrachten wir sowohl den allgemeinen Policy-Wandel in Deutschland, den wir mit Hilfe verschiedener aggregierter Indikatoren nachzeichnen, als auch den speziellen Fall der Erstunterbringung von Flüchtlingen, um potentielle pfadbildende Mechanismen identifizieren zu können. Schließlich diskutieren wir die empirischen Erkenntnisse vor dem Hintergrund der theoretischen Annahmen und wagen einen Blick über die deutsche Fallstudie hinaus. Ein Fazit schließt diesen Beitrag.

\section{Theoretischer Rahmen}

Die Frage des vorliegenden Beitrages, inwiefern es in Deutschland im Politikfeld der Inneren Sicherheit zu einem Policy-Wandel gekommen ist, der nicht mehr mit dem bisherigen „mittleren Weg“ (Schmidt I987; Wenzelburger/Staff 20I6) vereinbar ist, lässt sich nur dann plausibel beantworten, wenn vorher klare Erwartungen dahingehend gebildet werden, welche Mechanismen 
bestehende Pfade aus dem Gleichgewicht bringen und neue Pfadabhängigkeiten schaffen (dazu: Beyer 2005). Denn nicht jedes Ereignis qualifiziert sich als critical juncture (Collier/Collier I99I) - also als ein herausragendes Ereignis, das Policies tatsächlich von ihrem historischen Pfad abbringt und einen neuen Pfad beginnen lässt.

Collier und Collier arbeiten in ihrem bahnbrechenden Aufsatz von 199I zwei zentrale Kriterien heraus 3 , anhand derer critical junctures analysiert werden können. Zum einen ist es erforderlich, die neu geschaffenen Policies explizit mit dem vorherigen Status Quo zu vergleichen (antecedant system) (Collier/Collier I99I, 34). Und zum anderen muss die legacy spezifiziert werden, d.h. es muss nachgewiesen werden, wie in einem kritischen Moment der Geschichte neue Pfadabhängigkeiten geschaffen werden, die tatsächlich einen neuen Policy-Pfad begründen: „If the explanatory hypothesis - that is the hypothesized critical juncture did not produce the legacy - is false, then one would assert that it was not, in fact, a critical juncture" (Collier/Collier 199I, 30). Anders formuliert können herausragende externe Ereignisse Policies kurzfristig von ihren historischen Pfaden abbringen - jedoch zeigt erst die Analyse der Mechanismen, die ihrerseits neue Pfadabhängigkeiten schaffen, ob sich tatsächlich tiefgreifender Wandel vollzogen hat, der in einen neuen Policy-Pfad mündet (Pierson 2004, I35).

Welche Mechanismen der Pfadabhängigkeit sind nun in der Phase einer critical juncture theoretisch zu erwarten? Sydow et al. (2009) haben sich in ihrem Ansatz en détail mit dieser formation phase befasst, in der Mechanismen auftreten, die neue pfadabhängige Prozesse schaffen - die also durch ihre Struktur zu einem lock-in führen. Nach Sydow et al. lassen sich vier Mechanismen unterscheiden, welche die Formation neuer pfadabhängiger Prozesse begünstigen:

I. Koordinationseffekte, bei denen einmal festgelegte Regeln (z.B. Rechtsfahren im Straßenverkehr) die Koordinationskosten für alle Teilnehmer spürbar senken und daher zum lock-in des Pfades führen. Im Bereich staatlicher Policies entstehen solche Koordinationseffekte regelmäßig, wenn über neugeschaffene Institutionen oder Gesetze neue Spielregeln für alle festgelegt werden, die für Klarheit über Prozesse und Verfahren sorgen.

2. Komplementaritäten, bei denen die Entscheidung für eine bestimmte Alternative das Kosten-Nutzen-Verhältnis für eine andere Wahlent-

3 In ihrem Aufsatz stellen Collier und Collier insgesamt zehn Punkte vor, die bei der Analyse von critical junctures zu beachten sind. Bei gründlicher Lektüre lassen sich die zehn Punkte jedoch in die genannten zwei Kategorien einordnen (Punkte I- 5 behandeln Fragen der legacy, Punkte 5-Io Fragen des Vergleichs mit dem „antecedant system" sowie alternativer Erklärungen). scheidung beeinflusst, da sich beide Entscheidungen komplementär zueinander verhalten und der gemeinsame Nutzen höher liegt als die Summe der Nutzen beider Alternativen für sich genommen (z.B. sind im Varieties-of-Capitalism-Ansatz (Hall/Soskice 200I) unterschiedliche Sphären zueinander komplementär, da sie nur bei gleichem Koordinationsmodus zu optimaler Performanz führen).

3. Lerneffekte, die dadurch für Effizienzgewinne sorgen, dass eine bestimmte Handlung wiederholt wird und damit die Weiterverfolgung dieser einmal erlernten Routinen wahrscheinlich machen. Im Bereich der Policies sollten solche Effekte insbesondere bei der Implementation von Maßnahmen vor Ort entstehen.

4. Effekte adaptiver Erwartungen, bei denen sich Handlungen an Erwartungen anderer ausrichten und sich in einem zirkulären Prozess gegenseitig bestätigen (z.B. erwarten Manager, dass ihre Mitarbeiter sich an monetären Anreizen ausrichten, die Mitarbeiter verhalten sich entsprechend der Erwartungen und bestätigen damit die Erwartungen der Manager (McGregor 1960)). Wenn Policies über ihre Regelungen solche Erwartungsbildungen ermöglichen, sind auch solche Effekte zu erwarten.

Die Forschungsfrage dieses Beitrags steht und fällt also mit dem Nachweis dieser vier Effekte sowie mit dem Vergleich des neuen mit dem alten Status Quo. Der nächste Abschnitt präsentiert die empirische Evidenz zu unserem Fall entlang der Empfehlungen von Collier und Collier (199I).

\section{Empirische Evidenz}

Im Kontext der Flüchtlingskrise wurden viele sicherheitspolitische Entscheidungen auf unterschiedlichen Verwaltungsebenen des deutschen Staates getroffen, die sich nur schwer zur Gänze analysieren lassen. Um einerseits die Gesamtentwicklung (antecedent system) darzustellen, andererseits auch die Mechanismen (legacy) zu untersuchen, geht dieser Abschnitt in zwei Schritten vor. Zunächst stellen wir die Entwicklungen verschiedener aggregierter Maßzahlen über die Zeit mit einem besonderen Fokus auf die Entwicklung seit dem Sommer 2015 dar. Dabei unterscheiden wir zwischen Veränderungen, die die staatliche Bereitstellung von Sicherheit betreffen, und Entwicklungen im Markt privater Sicherheitsdienstleister. Um einen näheren Blick auf die möglicherweise pfadprägenden Mechanismen werfen zu können, fokussieren wir sodann in einem zweiten Schritt detailliert auf die Frage, wie „Sicherheit“ im Feld 
der Flüchtlingsunterbringung organisiert wurde, weil gerade dort besonders ertragreich die Auswirkungen der Flüchtlingskrise einerseits und die Veränderungen der Aufgabenteilung zwischen Staat und Markt analysiert werden können.

Die folgende empirische Analyse basiert daher auf unterschiedlichen Datenquellen. Erstens haben wir für die Aggregatdatenanalyse Haushaltspläne des Bundes, Daten zur Entwicklung des Polizeipersonals, die gesetzgeberischen Veränderungen des legislativen Status Quo im Bereich des Strafrechts und der Inneren Sicherheit und Zahlen zur Entwicklung des privaten Sicherheitsgewerbes ausgewertet. Zweitens haben wir für die detaillierte Analyse der sicherheitspolitischen Entscheidungen im Bereich der Flüchtlingsunterbringung im Jahr 2016 einen Fragebogen an alle Stellen in den Bundesländern verschickt, welche für die Gewährleistung von Sicherheit in den Erstaufnahmeeinrichtungen (EAE) der Länder zuständig sind. ${ }^{4}$ Leider haben trotz intensiven Nachfassens nur etwa zwei Drittel der angeschriebenen Behörden den Fragebogen beantwortet, weshalb in der empirischen Auswertung für einige Länder keine weiteren Angaben gemacht werden können. Durch diesen breiten Ausgriff in der Datengewinnung sind wir zuversichtlich, die wichtigsten sicherheitspolitischen Policy-Reaktionen auf die Flüchtlingskrise im Kernbereich der Unterbringung erfasst zu haben.

\subsection{Aggregatanalyse: Die Entwicklung des Politikfelds über die Zeit}

Um die Entwicklungen im Politikfeld zu beschreiben, unterscheiden wir in der folgenden Aggregatdatenanalyse zwischen staatlichen und privaten Akteuren. Eine Berücksichtigung von nur staatlicher Sicherheitsfürsorge wäre angesichts des starken Wachstums privater Sicherheitsdienstleistungen wenig zielführend. Unsere Analyse bezieht sich dabei sowohl auf Entwicklungen auf Länder- (z.B. hinsichtlich der Polizistenzahlen) als auch auf Bundesebene.

\subsubsection{Der Staat}

Drei Dimensionen des Politikfelds sind für die Darstellung von Veränderungen auf der Aggregatebene von besonderem Interesse: die Ausgaben für die allgemeine Sicherheitsproduktion im Bund, die in der politischen Debatte zentrale Maßzahl der Polizeistärken (d.h. die Zahl der Polizisten) (Tepe/Vanhuysse 2013) und - um über Budgetdaten hinaus tatsächliche Policy-Outputs in den Blick zu nehmen - die Bundesstraf- und Sicherheitsgesetzgebung.

4 Für wertvolle Unterstützung bei der Fragebogenerstellung danken wir Kathrin Hartmann.
Die Ausgaben für das Bundesministerium des Innern (BMI) und seinen Geschäftsbereich (Einzelplan 06) sind von 2014 bis 2017 deutlich angestiegen (BMI 2017). Die absoluten Zahlen (2014: 5.898.816.000 €/ 2017: 8.977.588.0oo $€$ ) belegen diese Steigerung um mehr als 3 Mrd. € deutlich. Damit erfährt das BMI ein überproportionales Wachstum seines Budgets im Vergleich zum Gesamthaushalt und steigert seinen Anteil an diesem von I,99\% im Jahr 2014 auf 2,73\% im Jahr 2017. Durch diese zusätzlichen Mittel wachsen in diesem Zeitraum die Budgets von Sicherheitsbehörden wie dem Bundesamt für Verfassungsschutz (+66,40\%), dem Bundeskriminalamt $(+38,10 \%)$ oder der Bundespolizei $(+31,71 \%)$ deutlich. Innerhalb des Einzelplans 06 nimmt die relative Bedeutung dieser Sicherheitsorgane aber (mit Ausnahme des Bundesamts für Verfassungsschutz) trotz der großen Ausgabensteigerungen ab. Diese Entwicklung steht im Zusammenhang mit dem Aufwuchs von Ausgaben für zwei Sachbereiche, die einen unmittelbaren Bezug zur Flüchtlingskrise aufweisen und dem Geschäftsbereich des BMI zugeordnet sind: So wächst der Anteil des Bundesamts für Migration und Flüchtlinge (BAMF) am Einzelplan o6 von 2,70\% im Jahr 2014 auf 8,71\% im Jahr 2017. Zusätzlich steigert sich der Anteil des Haushaltspostens „Integration und Migration, Minderheiten und Vertriebene" von 6,26\% auf 9,99\%. In absoluten Zahlen entsprechen diese Mehrausgaben über vier Jahre in beiden Bereichen zusammen genommen knapp I,I5 Mrd. $€$ und machen damit mehr als ein Drittel der Gesamtsteigerungen aus. Zusammenfassend kann also festgehalten werden, dass im Zeitraum der Flüchtlingskrise die Ausgaben für das Bundesministerium des Innern deutlich wachsen, was auch den Sicherheitsorganen zugute kommt. Allerdings muss diese Steigerung im BMI-Haushalt auch in Relation zum Wachstum des Gesamthaushalts und vor allen Dingen mit Rücksicht auf die hohen Ausgaben für Aufgaben im Bereich der Asylund Integrationspolitik interpretiert werden.

Relativ stabil entwickelten sich die Polizeistärken in den deutschen Bundesländern (Abbildung I). Grundsätzlich sinkt die Anzahl der Polizeibeamten pro Einwohner leicht, insbesondere in den östlichen Bundesländern, oder sie stagniert. Dies weist auf die bekannte Konstanz von Personalstärken hin, die in Deutschland durch den Beamtenstatus noch verstärkt wird. ${ }^{5}$ Größere Abweichungen vom Durchschnitt ergeben sich naturgemäß für die Stadtstaaten Berlin, Hamburg und Bremen. Ein besonders prägnanter Effekt der Flüchtlingskrise kann aus dieser Darstellung nicht abgeleitet werden allerdings weisen 2016 elf von I6 Bundesländern eine höhere Polizistenzahl auf als im Vorjahr.

5 Tepe und Vanhuysse (2013) finden in ihrer Regressionsanalyse der Bestimmungsfaktoren von Polizeistärken einen hochsignifikanten Effekt der Vorjahresbeschäftigung auf die Beschäftigung im Folgejahr. 
Abbildung 1: Entwicklungen der Polizistenzahlen in Deutschland, 1998-2016

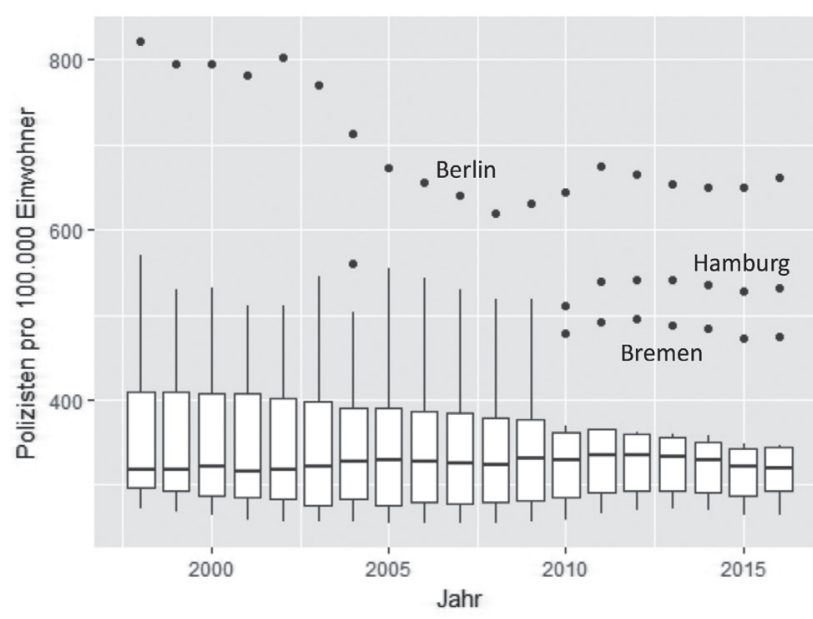

Anmerkungen: Quellen - Polizisten: Statistisches Bundesamt, Personal des öffentlichen Dienstes - Fachserie 14 Reihe 6, Tabellenblatt 7.5, Vollzeitäquivalente - Beamte und Arbeitnehmer; Bevölkerung: Statistisches Bundesamt Genesis, Abfrage, Fortschreibung des Bevölkerungsstandes, Bevölkerung: Bundesländer, Stichtag [für 2016, Bevölkerungszahlen vom 31.12.2015].

Ein etwas anderes Bild ergibt sich aus einer Analyse der parlamentarischen Haushaltsdebatten ${ }^{6}$ in den Ländern im Zeitraum Oktober 2015 bis Januar 2016. Sie lässt sowohl Rückschlüsse auf quantitative Veränderungen als auch auf die politische Begründung dieser zu. Auch wenn sich ein direkter Vergleich zwischen den Ländern alleine aufgrund der unterschiedlichen Haushaltsplanarten verbietet, ergibt die Auswertung der haushaltspolitischen Plenardebatten in den Ländern zu einer "HochZeit" der Flüchtlingskrise drei zentrale Ergebnisse:

I. In fast allen Bundesländern sehen die Haushaltspläne eine teilweise deutliche Erhöhung der Polizeistärken ${ }^{7}$ vor.

2. Zusätzlich zu den Stellenschaffungen bei den Landespolizeien stocken verschiedene Länder sogenannte (geringer qualifizierte) Wachpolizisten auf (Hessen, Berlin) bzw. führen diese ein (Saarland, Sachsen, Sachsen-Anhalt; im Saar-

6 In den Fällen, in denen ein direkter Rückschluss auf konkrete Zahlen aus dem Plenarprotokoll nicht möglich war, wurde auf sekundäre Quellen zurückgegriffen. Da nur im Fall Brandenburgs die Haushaltsdebatte im Januar 2016 stattfand und Haushaltspläne einen längeren Vorlauf aufweisen, werden Effekte der Ereignisse der Silvesternacht 2015/2016 in Köln ausgeschlossen. In den Länderparlamenten von Hamburg, Bremen und Sachsen wurden in diesem Zeitraum keine Haushaltsdebatten geführt, weshalb sie aus der Untersuchung ausgeklammert wurden.

7 Hier ausgeklammert aber dennoch erwähnenswert sind neben den personellen Aufstockungen auch die zeitgleichen generellen finanziellen Anstrengungen der Länder im Sicherheitsbereich wie z.B. beim Verfassungsschutz (Baden-Württemberg), bei der Polizeiausstattung (Bayern, Thüringen) oder in Form von Stellenhebungen (Hessen). land mit direktem Verweis auf den „Objektschutz" also zur Bewachung von Aufnahmeeinrichtungen) (Mitteldeutscher Rundfunk 2015). ${ }^{8}$

3. Die Herausforderungen der Flüchtlingskrise stellen einen dominanten oder gleichberechtigten Argumentationsstrang für die Begründung des Aufwuchses im Bereich der Polizei dar. Nur in zwei der I 3 untersuchten Debatten werden die personellen Aufstockungen ausschließlich mit allgemeinen kriminalistischen Anforderungen und insbesondere der Gefahr durch Terrorismus begründet.

Auf Bundesebene ergeben die Haushaltsberatungen ${ }^{9}$ zum Einzelplan o6 (BMI) im Haushaltsplan 2016 ein ähnliches Bild, wobei insbesondere die Aufstockung des BKA um 317 und der Bundespolizei um I.568 Stellen für 2016 (insgesamt 3.000 Stellen bis 2018) hervorstechen (Schuster 2015). Wie in den Ländern wird der personelle Zuwachs auf der Bundesebene vor allem mit den Anforderungen der Flüchtlingskrise ${ }^{\mathrm{IO}}$ aber auch mit Terrorgefahren begründet. ${ }^{I I}$

Die Entwicklung der Gesetzgebung im Straf- und Sicherheitsrecht soll nun nach der Darstellung von Personal- und Budgetdaten eine dritte Dimension von staatlicher Sicherheitsgestaltung darstellen. Wir folgen hier der Empfehlung von Knill und Tosun (2O12, 229), die darauf hinweisen, dass die direkte Messung von "policies" eine genauere Analyse erlaubt als OutcomeIndikatoren (wie z.B. Gefangenenraten). Basierend auf der Messung und dem Kodierschema von Wenzelburger und Staff (2016), dem alle Gesetzesentscheidungen des Bundes im Bereich des Straf- und Sicherheitsrechts seit 1993 zugrunde liegen, können wir die monatliche Nettoveränderung in der Schärfe der Gesetzgebung darstellen (siehe Abbildung 2).

Der für uns in diesem Kontext interessante Zeitraum der dritten Regierung Merkel von 2013 bis 2017 fällt im Vergleich zu anderen Legislaturperioden sowohl durch einen massiven Ausschlag im ersten Halbjahr 2017 als auch durch eine gewisse Masse und Dichte von verschärfenden gesetzgeberischen Maßnahmen auf. Der Anfang dieses Zeitraumes, in dem mehr und eher verschärfende Gesetze beschlossen wurden, koinzidiert mit der Flüchtlingskrise und ihrem weiteren Verlauf. Tatsächlich zeigt sich in der Krise und nachfolgend eine weitgehende politische Verkopplung des Politikfelds der Inneren Sicherheit und des der Migration, sodass Gesetzesänderungen im Feld der Asylpolitik mit sicherheitsbezogenen Regelungen aufgeladen werden und andersherum dazu auch: Wenzelburger/Staff 2018.

\footnotetext{
8 Saarland, Landtagsplenarprotokoll I5/43: 3725 .

9 Bundestagsplenarprotokoll I8/138: I3540-13560.

Io Bundestag, Plenarprotokoll I8/I38: 13555.

II Bundestag, Plenarprotokoll I8/138: I3547.
} 
Abbildung 2: Monatliche Nettoveränderung in der Schärfe der deutschen Straf- und Sicherheitsgesetzgebung, 1993-2017

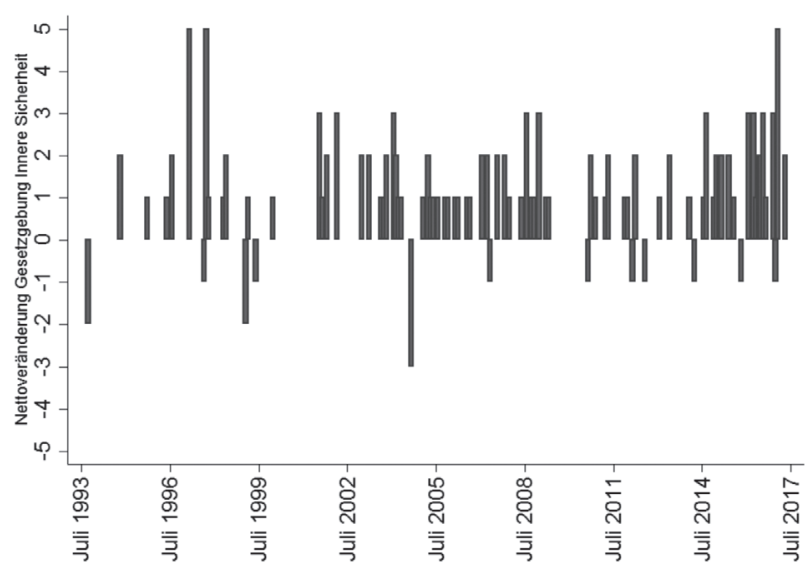

Anmerkungen: Quelle und Kodierschema - Wenzelburger/Staff (2016); Negative Werte entsprechen einer Liberalisierung, positive Werte einer Verschärfung.

Ausgeklammert aus dieser Betrachtung von Aggregatdaten öffentlicher Sicherheitsproduktion bleiben die deutschen Streitkräfte, die bis auf wenige Ausnahmen nicht im Inland eingesetzt werden dürfen (Art. 87a Abs. 2 GG). Eine dieser Ausnahmen ist die Amtshilfe, in deren Rahmen die Bundeswehr in der Flüchtlingskrise umfangreiche Unterstützungsleistungen in den Bereichen Transport, Unterbringung, Versorgung und Personal für Länder, Kommunen und das Bundesamt für Migration und Flüchtlinge (BAMF) erbrachte..$^{12}$ Auch im Unterschied zu Österreich, wo das Bundesheer nicht nur unterstützende, sondern auch sichernde bzw. ordnende Aufgaben wahrgenommen hat (Bundesheer 2016), haben die deutschen Streitkräfte damit aber keine direkten sicherheitsrelevanten Tätigkeiten übernommen.

\subsubsection{Der Markt}

Nun wenden wir uns der privaten Sicherheit zu. Ein Blick auf die Entwicklung der Mitarbeiter- und Umsatzzahlen der privaten Sicherheitsbranche in Deutschland (siehe Abbildung 3) zeigt zunächst die grundsätzliche und gleichmäßige Tendenz eines stabilen Wachstums - mit einer gewissen Abflachung zwischen 2008 und 20I0. Im internationalen Vergleich sind der Stand und die Entwicklung der Branche jedoch eher moderat (Van Steden/De Waard 2013; Van Steden/Sarre 2007).

In dem für uns besonders interessanten Jahr 2015 zeigt sich jedoch ein deutlicher Wachstumsschub sowohl im Hinblick auf die Mitarbeiter als auch die Umsätze der Branche. Dieser setzt sich (insbesondere bzgl. des Umsatzes) im Jahr 2016 fort. Ein Zusammenhang mit der

I2 Bundestag, Drucksache 18/9599.
Abbildung 3: Mitarbeiter und Umsatz der privaten Sicherheitsbranche in Deutschland, 1998-2016

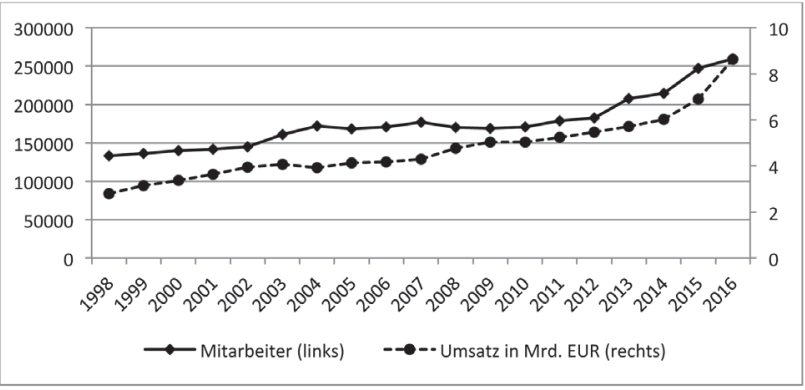

Anmerkungen: Quelle - BDSW/BDGW (2017).

Abbildung 4: Entwicklung der Beschäftigten und der freien Stellen bei privaten Wach- und Sicherheitsdiensten in Deutschland, 2013-2017

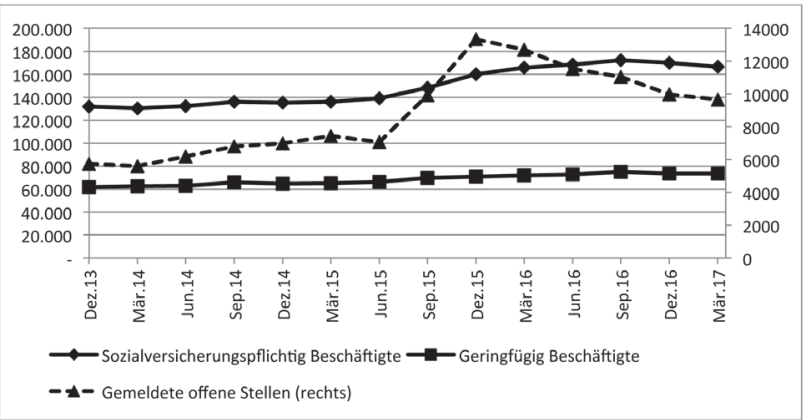

Anmerkungen: Quellen - Bundesagentur für Arbeit, Reihe „Gemeldete Arbeitsstellen nach Wirtschaftszweigen“, Tabellenblatt 9, Kategorie 801 („Private Wach- und Sicherheitsdienste" ) / Bundesagentur für Arbeit, Reihe „Beschäftigte nach Wirtschaftszweigen“, Tabellenblätter 1 und 3, Kategorie 801 („Private Wach- und Sicherheitsdienste“).

Flüchtlingskrise durch einen Boom bei Bewachungsaufträgen für Aufnahmeeinrichtung ist sehr wahrscheinlich (siehe unten).

Noch deutlicher zeigt sich dieser Zusammenhang in den monatlichen Zahlen der von den Sicherheitsunternehmen an die Arbeitsagenturen zur Vermittlung gemeldeten offenen Arbeitsstellen (siehe Abbildung 4). Die offenen Stellen verdoppeln sich zwischen August 2014 und Januar 2016 und liegen damit deutlich über dem allgemeinen Wachstum im Dienstleistungssektor im selben Zeitraum. Dass die Flüchtlingskrise diese Entwicklung erklären kann, liegt nicht nur wegen der zeitlichen Koinzidenz und der direkten Abnahme offener Stellen nach dem Höhepunkt der Zuwanderung auf der Hand: Denn die meisten offenen Stellen wurden in den Ländern gemeldet, die nach dem Königsteiner Schlüssel eine höhere Anzahl von Flüchtlingen aufnehmen sollten oder mehr Personen aufgenommen haben. Auch wenn gemeldete offene Stellen keinen direkten Schluss auf die tatsächliche Größe der Industrie zulassen, so ergibt sich doch das klare Bild einer plötzlich wachsenden Industrie mit stark steigendem Personalbedarf. Der Rückgang der 
Zahl der offenen Stellen gegen Ende der Beobachtungsperiode weist indes keinesfalls auf ein Schrumpfen des privaten Sektors hin, sondern zeigt allenfalls, dass durch die in Gang gesetzten Maßnahmen im Bereich der Ausbildung die Überschussnachfrage im Markt zumindest teilweise gedeckt werden konnte.

Den steigenden Trend der Beschäftigung in diesem Sektor weisen auch die Quartalszahlen der Beschäftigungsstatistik der Bundesagentur für Arbeit (2OI7) nach. So waren im März 20I5 etwa 200.00o Beschäftigte (sozialversicherungspflichtig und geringfügig) bei „privaten Wach- und Sicherheitsdiensten“ tätig, ein Jahr später waren es rund 40.000 Beschäftigte mehr. Besondere Beachtung verdient dabei der Aspekt, dass die Beschäftigungszahlen trotz des Rückgangs der Flüchtlingszahlen bis in den September 2016 weiterhin wuchsen. Eine Beobachtung, die auch den Branchenverband BDSW (2017) überrascht, der ein anhaltendes „Schutzbedürfnis“ bei "Wirtschaft, Staat, Kommunen und - zu einem kleinen Teil - auch [bei] privaten Haushalten" feststellt. Erst im Dezember 2016 gehen die Zahlen etwas zurück, bleiben jedoch weiterhin auf hohem Niveau und deutlich über dem Vorkrisenstand.

Insgesamt bestätigen die Zahlen dieser Aggregatanalyse also den Befund, dass während und nach der Flüchtlingskrise ein doppelter Sicherheitsaufwuchs stattgefunden hat. Zum einen gibt der Staat mehr Geld im Politikfeld aus, beabsichtigt bei Bund und Ländern mehr Personaleinstellungen und verschärft deutlich die Strafund Sicherheitsgesetzgebung. Darüber hinaus liegt, zum anderen, aber auch ein Zusammenhang zwischen der Flüchtlingskrise und dem starken Wachstum der privaten Sicherheitsbranche im gleichen Zeitraum nahe. Die Zahlen belegen deutlich, wie Umsätze und Mitarbeiterzahlen wachsen und jedenfalls bis ins Frühjahr 2017 nicht auf ein Vorkrisenniveau zurückfallen. Was diese Zahlen aber nicht darstellen können, ist, inwieweit der gezeigte Zusammenhang auch von den eingangs theoretisierten kausalen Mechanismen und Wechselwirkungen der Pfadabhängigkeit nachhaltig gestützt wird. Dieses soll im Folgenden mit Blick auf einen speziellen Fall von Sicherheitsorganisation in der Flüchtlingskrise geschehen.

\subsection{Pfadformierende Mechanismen in der Flücht- lingsunterbringung}

Der Fall der Flüchtlingsunterbringung eignet sich besonders gut für die Untersuchung von möglichen pfadformierenden Mechanismen, da die Frage der Unterbringung der Geflüchteten ganz konkrete sicherheitspolitische Herausforderungen mit sich brachte, die von den politisch Verantwortlichen adressiert werden mussten. Im Folgenden zeigen wir anhand der Ergebnisse aus einer qualitativen Befragung der Aufsichtsbehörden der Länder zu den operativen Entscheidungen im Zu- sammenhang mit Erstaufnahmeeinrichtungen mittels eines Fragebogens, welche sicherheitspolitischen Entscheidungen im Kontext der Flüchtlingsunterbringung gefallen sind und identifizieren auf Basis dieser Evidenz pfadformierende Mechanismen.

Neben Schritten zur Reform der grundsätzlichen Regulierung des Bewachungsgewerbes auf Bundesebene, wurden in den Bundesländern eigene Regelungen zur Vergabe von Aufträgen, zur Ausgestaltung dieser Aufträge und zur Kontrolle der zu erbringenden Dienstleistungen geschaffen (Tabelle I). Die schiere Zahl der unterzubringenden Flüchtlinge und Berichte über Qualitätsmängel beim eingesetzten privaten Bewachungspersonal setzten dabei die Behörden der Länder unter starken Druck, neue - zumeist verschärfte - Standards für den Einsatz privater Sicherheitsdienstleister zu formulieren. Dabei gleicht der aktuelle Regulierungsstand einem Flickenteppich, der sich durch die schnelle und an der konkreten Situation orientierte Reaktion der für den Einsatz privater Sicherheitskräfte zuständigen Landesbehörden ergibt. Denn im Bereich der Vergabe von Aufträgen an private Sicherheitsdienstleister müssen sich die Behörden in den Ländern zwar am allgemeinen Vergaberecht orientieren, setzen aber durchaus eigene Schwerpunkte. So erließ etwa Nordrhein-Westfalen bereits 2014 eigene Standards zur Vergabe als direkte Reaktion auf eine Reihe von Skandalen in Aufnahmeeinrichtungen des Landes. Andere Länder gingen dazu über, von den Firmen bestimmte Konzepte einzufordern, die sich mit Qualitätsmanagement und Fortbildung (Brandenburg), Personalqualität und Dienstleistungsqualität (SchleswigHolstein) oder Personaleinsatz, Personalkompetenz und Reaktionen auf Belegungszahlen (Regierungspräsidium Freiburg) beschäftigen..$^{13}$ Die Laufzeiten der Verträge mit den zur Bewachung eingesetzten Sicherheitsfirmen sind in den Ländern unterschiedlich und liegen zwischen ein bis sieben Jahren. Nordrhein-Westfalen, Sachsen und das Regierungspräsidium Tübingen (Baden-Württemberg) berichteten Ausstiegsklauseln bei Schließung der EAE, bzw. dass grundsätzlich die Laufzeit an den Betrieb der EAE gekoppelt ist. Auch die Aufsicht der Länder über die in den EAEs eingesetzten privaten Sicherheitsfirmen wurde in Reaktion auf Berichte über Qualitätsmängel diskutiert. Einige Länder, etwa die Regierungspräsidien Freiburg und Tübingen (Baden-Württemberg) verlangen daher die Vorlage von Führungszeugnissen oder die Überprüfung durch die Einrichtungsleitung und bestellte Sicherheitsberater. Die Kontrolle des eingesetzten Sicherheitspersonals durch Abfrage bei Sicherheitsbehörden spielt ebenfalls eine zentrale Rolle und nahm die

I3 Schleswig-Holstein schreibt zudem vor der Prüfung dieser Unterlagen als formale Voraussetzung eine Sachkundeprüfung, eine ErsteHilfe- und Brandschutzhelferausbildung, ein Jahr Berufserfahrung sowie die Überprüfung durch das Landeskriminalamt vor - ein im Vergleich außergewöhnlich hoher Qualitätsanspruch. 
Tabelle 1: Regulierung privater Sicherheitskräfte in EAEs

\begin{tabular}{|c|c|c|c|}
\hline Land & Vergabekonzept & Laufzeit & Weiterführende Aufsicht \\
\hline BW Fr & $\begin{array}{l}\text { Qualitätskonzepte } \\
\text { (40\% Preis, } 60 \text { \% Konzept) }\end{array}$ & $1 \mathrm{Jahr}$ (+ optional $1 \mathrm{Jahr}$ ) & Keine \\
\hline BW Ka & Keines & 1-3 Jahre & Keine \\
\hline BW St & Keines & 2 Jahre & k.A. \\
\hline BW Tü & k.A. & $\begin{array}{l}\text { Abhängig von EAE (optionale } \\
\text { Kündigung bei Schließung } \\
\text { der EAE) }\end{array}$ & Kontrollen vor Ort (Sicherheitsberater) \\
\hline BB & Qualitätskonzepte & 3 Jahre (+ optional 2 Jahre) & $\begin{array}{l}\text { Kontrollen vor Ort, Personalabfragen bei } \\
\text { Sicherheitsbehörde }\end{array}$ \\
\hline MV & Keines & 2-3 Jahre (+ optional 1 Jahr) & Kontrollen vor Ort \\
\hline $\mathrm{NI}$ & In Überarbeitung & 1-7 Jahre & k.A. \\
\hline NW & Personelle Anforderungen & $\begin{array}{l}\text { Abhängig von Laufzeit der } \\
\text { EAE }\end{array}$ & $\begin{array}{l}\text { Kontrollen vor Ort, Personalabfragen bei } \\
\text { Sicherheitsbehörde }\end{array}$ \\
\hline RP & Keine & 1 Jahr (im Durchschnitt) & Personalabfragen bei Sicherheitsbehörde \\
\hline $\mathrm{SH}$ & $\begin{array}{l}\text { Qualitätskonzepte, } \\
\text { personelle Anforderungen } \\
\text { (60\% Preis, } 40 \% \text { Konzept) }\end{array}$ & $\begin{array}{l}\text { Abhängig von EAE } \\
\text { (Mietdauer) }\end{array}$ & $\begin{array}{l}\text { Kontrollen vor Ort, Personalabfragen bei } \\
\text { Sicherheitsbehörde }\end{array}$ \\
\hline SL & k.A. & k.A. & k.A. \\
\hline SN & Personelle Anforderungen & $\begin{array}{l}1 \text { Jahr (optionale Kündigung } \\
\text { bei Schließung der EAE) }\end{array}$ & Kontrollen vor Ort \\
\hline
\end{tabular}

Anmerkungen: Abkürzungen: BW Fr: Baden-Württemberg, Regierungspräsidium Freiburg; BW Ka: Baden-Württemberg, Regierungspräsidium Karlsruhe; BW St: Baden-Württemberg, Regierungspräsidium Stuttgart; BW Tü: Baden-Württemberg, Regierungspräsidium Tübingen; k.A.: keine Angabe. Stand 2016.

oben angeführten Änderungen im Bewachungsrecht auf Bundesebene vorweg. So berichten Rheinland-Pfalz und Schleswig-Holstein von Überprüfungen durch das Landeskriminalamt sowie Brandenburg durch den Verfassungsschutz. Mecklenburg-Vorpommern, NordrheinWestfalen, Schleswig-Holstein und Sachsen erwähnen ferner direkte Kontrollen in der EAE durch die aufsichtführende Stelle. Insgesamt zeigen die Antworten der Länderbehörden, dass eine Sensibilisierung für die Regulierung und Qualitätssicherung der privaten Sicherheitsdienstleister nach den Skandalen in einigen EAEs erfolgte. In vielen Ländern führten diese zur Ausarbeitung einheitlicher Standards und Qualitätskonzepte, die sowohl bei der Ausschreibung von Verträgen als auch im Betrieb selbst zum Einsatz kommen.

Unter dem Strich lassen die Auskünfte aus den Landesbehörden darauf schließen, dass Fragen der Vergabe, Aufsicht oder Kontrolle privater Sicherheitsdienstleister in den Ländern anscheinend häufig erstmalig im Zuge der Flüchtlingsunterbringung umfassend geregelt worden sind.

Eine weitere zentrale Herausforderung im Zusammenhang mit der Gewährleistung von Sicherheit bei der
Flüchtlingsunterbringung betrifft das Verhältnis zwischen privaten und staatlichen Akteuren. Denn wenn Landesbehörden (und auch Kommunen) - wie geschehen stark auf private Sicherheitsdienstleister zurückgreifen, ${ }^{14}$ stellt sich die Frage, wie diese privaten Akteure mit staatlichen Akteuren zusammenarbeiten. Aus den Antworten in den Fragebögen wird eine klare Aufgabenteilung zwischen einfachen Sicherheitsaufgaben, die von privaten Dienstleistern durchgeführt werden, und umfangreicheren Aufgaben deutlich, die von der Polizei übernommen werden (Tabelle 2). Rückmeldungen aus dem Saarland, dem Regierungspräsidium Karlsruhe (Baden-Württemberg) und Schleswig-Holstein illustrieren die permanente Präsenz der Landespolizei in den EAEs: In Schleswig-Holstein sieht etwa ein landesweites Konzept die Präsenz von fünf Beamten ab ca. 500 Flüchtlingen vor, wobei in größe-

I4 Der Aufwuchs im Bereich der privaten Sicherheit in den EAEs ist in manchen Ländern außerordentlich stark. Nordrhein-Westfalen - das Bundesland mit dem höchsten Aufnahmeschlüssel - beschäftigte mittelbar im vierten Quartal 20I4 ca. 200 private Sicherheitskräfte in den Flüchtlingseinrichtungen in Landeszuständigkeit. Diese Zahl verdoppelte sich im ersten Quartal 2015 und stieg bis zum vierten Quartal desselben Jahres auf 5.500 an. Im Sommer 2016 lag die Zahl bei ca. 6.600 . 
ren EAEs aber auch lageangepasst bis zu 20 Polizisten vor Ort sein können. Die Aufgabenbereiche der eingesetzten Polizisten, wie sie zum Beispiel für den Fall SchleswigHolsteins geschildert wurden, decken sich nur teilweise mit den Dienstleistungen des privaten Personals; insbesondere besitzt die Polizei eine Ermittlungsfunktion und -kompetenz, die den privaten Wachleuten fehlt. Insgesamt bestätigt sich das Bild der Literatur zum Einsatz privater Sicherheitskräfte (Kirsch 2003; Wakefield 2003, I65-192; Van Steden 2007; Löfstrand et al. 2016): Sie werden vor allen Dingen zur Einlasskontrolle und zu Ordnungszwecken eingesetzt und dienen durch ihre Präsenz der direkten Weiterleitung von Informationen an andere Stellen, die dann letztendlich die Lage bearbeiten. Ferner übernimmt das Wachpersonal auch sicherheitsferne und eher allgemeine Hausmeister-, Ansprech- und Informationsfunktionen.

Zusammenfassend lässt sich also für die Entscheidungen auf Ebene der Landesbehörden festhalten, dass die Herausforderungen der Flüchtlingskrise dazu führten, dass häufig zum ersten Mal umfassend Fragen der Vertragsgestaltung, Aufsicht und Kontrolle von privaten Sicherheitsunternehmen geregelt wurden. Daneben kam es auch zu einer Abgrenzung der Aufgaben privater Akteure von denen staatlicher Akteure, wobei die privaten Dienstleister häufig für „einfachere“ Sicherheits- dienste eingesetzt wurden, sich also eine Art Komplementarität der Aufgaben zwischen staatlichen und privaten Sicherheitsakteuren einstellte.

\section{Diskussion: Die Flüchtlingskrise als critical juncture?}

In diesem abschließenden Kapitel diskutieren wir nun, wie auf Basis der hier präsentierten Evidenz die eingangs gestellte Frage beantwortet werden kann, inwiefern die im Kontext der Flüchtlingsaufnahme beschriebenen Veränderungen in der Sicherheitspolitik in Deutschland den bestehenden Policy-Pfad im Politikfeld der Inneren Sicherheit durchbrochen haben. Hierzu greifen wir auf die oben dargestellten Überlegungen von Collier und Collier (I99I) zur Feststellung von critical junctures sowie von Sydow et al. (2009) zu Mechanismen der Pfadabhängigkeit zurück.

Collier und Collier (I99I, 34) nennen als erstes Kriterium zur Identifikation einer critical juncture, dass für einen Pfadbruch eine Differenz zwischen antecedent system und dem Status Quo sichtbar sein muss. Die Beschreibung des Politikfelds der Inneren Sicherheit im Schatten der „Flüchtlingskrise“ hat die deutlichen Veränderungen in den vergangenen drei Jahren nachgewiesen. Kenn-

Tabelle 2: Aufgaben privater und öffentlicher Sicherheitskräfte in EAE

\begin{tabular}{|c|c|c|c|}
\hline Land & Dienstleistungen & Sonst. Dienstleistungen & Rolle der Polizei \\
\hline BW Fr & Sicherheit, Ordnung & Keine & k.A. \\
\hline BW Ka & $\begin{array}{l}\text { Sicherheit, Ordnung, } \\
\text { Gefahrenabwehr }\end{array}$ & Kernbetrieb, Hausmeister & Feste Präsenz (3-9 Beamte) \\
\hline BW St & k.A. & k.A. & k.A. \\
\hline BW Tü & $\begin{array}{l}\text { Sicherheit, Ordnung, } \\
\text { Gefahrenabwehr, Präsenz }\end{array}$ & Hausmeister & Nur vereinzelte feste Präsenz \\
\hline BB & $\begin{array}{l}\text { Sicherheit, Ordnung, } \\
\text { Gefahrenabwehr }\end{array}$ & Kernbetrieb & Zeitliche Präsenz (2 Beamte) \\
\hline MV & Sicherheit, Ordnung & Keine & Keine feste Präsenz \\
\hline $\mathrm{NI}$ & In Überarbeitung & Hausmeister, Kernbetrieb & k.A. \\
\hline NW & $\begin{array}{l}\text { Sicherheit, Ordnung, } \\
\text { Gefahrenabwehr }\end{array}$ & Keine & Keine feste aber zeitliche Präsenz \\
\hline RP & Sicherheit, Ordnung & Keine & Personalabfragen bei Sicherheitsbehörde \\
\hline $\mathrm{SH}$ & $\begin{array}{l}\text { Sicherheit, Ordnung, } \\
\text { Präsenz }\end{array}$ & Hausmeister, Kernbetrieb & Räumliche Präsenz (5-20 Beamte) \\
\hline SL & Sicherheit, Ordnung & Keine & Räumliche Präsenz (2 Beamte) \\
\hline SN & Sicherheit, Ordnung & Kernbetrieb & Keine feste aber zeitliche Präsenz \\
\hline
\end{tabular}


zeichnend ist ein doppelter Aufwuchs beim Sicherheitspersonal: Sowohl Bundes-, Länder- und Hilfspolizeien wie auch - ganz besonders - die privaten Wachdienste wurden stark aufgestockt. Darüber hinaus reagierte der Bund mit Ausgabensteigerungen und einer deutlich verschärften Sicherheits- und Strafgesetzgebung sowie Regelungen, um die Qualität des Einsatzes privater Sicherheitsdienste in Flüchtlingsheimen zu gewährleisten. Auf Bundesebene ist hierzu 2016 auch ein entsprechendes Gesetz (Gesetz zur Änderung bewachungsrechtlicher Vorschriften) beschlossen worden. Insgesamt zeichnet unsere Analyse also das Bild eines sich im Wandel befindlichen Politikfelds, das sowohl von einem starken Ausbau des Sicherheitsapparates (staatlich und privat) als auch von lebhaften legislativen Aktivitäten gekennzeichnet ist.

Grundsätzliche - und in der kriminologischen Literatur bereits seit den I980er Jahren bekannte - Treiber der Privatisierung von Sicherheit wie die Zunahme privatisierter öffentlicher Räume (Shearing/Stenning I98I) oder ein erhöhtes gesellschaftliches Sicherheitsbedürfnis in Zeiten knapper öffentlicher Mittel (Bowden 1978; Forst 1999) ließen aber auch die deutsche Sicherheitsbranche vor 2015 wachsen. So nimmt die Branche bereits seit Jahrzehnten eine immer wichtigere Rolle in der Inneren Sicherheit ein. Jedoch lässt sich mit Ausnahme von Wiedervereinigungseffekten in den frühen I990er Jahren kein vergleichbar sprunghaftes Wachstum nachweisen (Hirschmann 2016, I35). „Selbst solch kritische Ereignisse wie der [sic!] des II. Septembers 200I haben im bundesdeutschen Raum keine großen quantitativen Bewegungen ausgelöst" (Hirschmann 2016, 135). Mit Blick auf die These der critical juncture scheint damit zumindest die Grundvoraussetzung erfüllt, dass ab 2015 eine Krise (oder alternativ eine "cleavage" (Collier/Collier I99I, 30)) vorliegt, die sich stark vom vorherigen Status Quo unterscheidet und Policy-Wandel ermöglicht.

Die zweite notwendige Bedingung, um von einer critical juncture sprechen zu können, ist jedoch die Formierung eines neuen Policy-Pfades, einer distinct legacy (Collier/Collier I99I, 24), welche zukünftige PolicyEntscheidungen beeinflusst. Entsprechend ist die entscheidende Frage, welche Mechanismen sich in den dargestellten Entwicklungen verbergen, die eine solche pfadformierende Wirkung entfalten können. Freilich ist die abschließende Klärung dieser Frage - wie so häufig in der Forschung zu Pfadabhängigkeit nur mit weiterem zeitlichen Abstand möglich; jedoch geben die bereits getroffenen Entscheidungen einen Hinweis auf zukünftige legacies, die mit Hilfe der von Sydow et al. (2009) freigelegten Mechanismen auf ihre pfadformierende Wirkung hin eingeschätzt werden können.

I. So kann von einem starken Koordinationseffekt ausgegangen werden, der sich aus der flächende- ckenden Anstellung privater Sicherheitsdienste für die Bewachung von EAEs in allen untersuchten Ländern ergibt. Dieser operative Konsens verringert für die Länder die zu befürchtenden politischen Kosten, die sich aus einer größeren politischen Diskussion um die Rolle privater Sicherheitsdienste sowie den damit verbundenen Skandalen (z.B. Misshandlungen von Flüchtlingen durch Sicherheitskräfte) ergeben könnten. Zudem werden die Koordinationskosten über neue bundesgesetzliche Regelungen reduziert, die für Klarheit über Prozesse und Verfahren sorgen - etwa mit Blick auf die Kontrolle des eingesetzten privaten Sicherheitspersonals. Die Behörden der Länder können durch die während der Flüchtlingskrise entwickelten Regeln für die Einbindung privater Dienstleister auch in Zukunft mit sehr viel geringeren Koordinationskosten auf private Sicherheitsdienstleister zugreifen. Denn sowohl die rechtlichen Rahmenbedingungen auf Bundesebene als auch die Prozesse in den Landesbehörden sind neu geregelt. Konkreter: Sollte in Zukunft für eine Großveranstaltung oder eine unvorhergesehene Einsatzlage privates Sicherheitspersonal notwendig sein, können Landesverwaltungen auf die nun festgelegten Vergabe- und Qualitätsstandards zurückgreifen und so ein höheres Vertrauen in die eingesetzten Mitarbeiter setzen, was die Koordinationskosten senkt und die Chance eines Einsatzes privater Sicherheitsdienste erhöht.

2. Im Hinblick auf Komplementaritäten dürfte die zumindest in einigen Bundesländern praktizierte engere Verknüpfung von privatem und staatlichem Sicherheitspersonal zum Schutz desselben Objektes Synergieeffekte erzeugen, die sich aus den unterschiedlichen Qualifikationsgraden ergeben. Arbeitsteilung im Bereich der Sicherheit - zwischen Landespolizeien, Hilfspolizeien und privaten Sicherheitsfirmen - wurde während der Flüchtlingskrise in vielen Ländern in viel größerem Ausmaß umgesetzt. Es ist zu erwarten, dass die einmal gemachten Erfahrungen und die sich daraus ergebenden Komplementaritäten zu einem lock-in dieses Policy-Pfads führen werden, der sich durch eine stärkere Arbeitsteilung im Bereich der Herstellung von Sicherheit auszeichnet.

3. Hinsichtlich regulativer Entscheidungen ist ferner von Lerneffekten auszugehen, die sich durch die konkrete Einbindung von privaten Sicherheitsdiensten in den EAEs und in der Zusammenarbeit zwischen Polizei und privaten Dienstleistern ergeben. Die Ergebnisse der Umfrage in den Länderbehörden lassen darauf 
schließen, dass in der täglichen Arbeit in den EAEs die unterschiedlichen Akteure ständig zusammenarbeiten (müssen), sodass entsprechende Abläufe zu Routinen werden. Genau diese Lerneffekte führen jedoch dazu, dass einmal eingeschlagene Policy-Pfade - zum Beispiel die massive Einbindung privater Sicherheitsdienstleistern zur Herstellung von Sicherheit in EAEs - auch in Zukunft fortgeführt werden.

4. Daneben ist mit Blick auf adaptive Erwartungen davon auszugehen, dass das Auftreten des Staates als Großkunde privater Sicherheitsdienstleistungen bei allen beteiligten Akteuren dazu führt, dass sich Erwartungen an die Herstellung von Sicherheit verändern und einander anpassen. Studien aus England (White 20IO) und Spanien (Gimenez-Salinas 2004) folgend dürfte der Einsatz privater Sicherheitsunternehmen durch den Staat sowie eine weiterführende Regulierung des Gewerbes auch zu einer größeren allgemeinen gesellschaftlichen Akzeptanz privater Sicherheitsdienstleistungen führen. Smith und White $(2014,426)$ fassen diesen Effekt so zusammen: „So while on one side regulation is used to 'fix' the market for security by imposing minimum standards on contractor operations (public protection), on the other it is used to 'camouflage' the market by shrouding these operations with reassuring symbols and structures of stateness (normative legitimation) (...) Regulation equates to legitimacy, and legitimacy enables contractors to sell their products and services more effectively". ${ }^{15}$

\section{Zusammenfassung und Fazit}

Der vorliegende Beitrag hat die Fragen aufgeworfen, I) welche sicherheitspolitischen Reaktionen die Flüchtlingskrise in Deutschland hervorgerufen hat und 2) inwieweit diese einen Pfadbruch darstellen. Die hier präsentierten empirischen Befunde weisen - mit Blick auf die erste Frage - darauf hin, dass ein doppelter Aufwuchs im Bereich des Sicherheitsapparates stattgefunden hat, der sowohl den Staat (die Polizeien) als auch den Markt (private Sicherheitsunternehmen) betrifft. Analysiert man diese Veränderungen im Hinblick auf

I5 Gegen einen solchen lock-in der Rolle von privatem Personal in der staatlichen Sicherheitsvorsorge sprechen freilich die teilweise sehr geringen oder vom Betrieb der Einrichtungen abhängigen Laufzeiten der Verträge mit den Sicherheitsunternehmen. Allerdings weisen aus unserer Sicht die dargestellten Mechanismen ebenso wie die weiterhin steigenden Beschäftigungszahlen der Branche darauf hin, dass private Sicherheit auch in Zukunft ein wichtigerer Baustein in der Herstellung öffentlicher Sicherheit sein wird, als das bisher der Fall war. ihre Qualität, so deuten die Ergebnisse - im Sinne der zweiten Forschungsfrage - darauf hin, dass das Politikfeld der Inneren Sicherheit im Kontext der Flüchtlingskrise tatsächlich einen Pfadbruch und den Beginn eines neuen Policy-Pfads erlebt hat. Den Ansätzen von Collier und Collier (199I) und Sydow et al. (2009) folgend haben wir herausgearbeitet, dass sowohl die Veränderungen in der Regulierung auf Bundesebene als auch die vielen kleinen Entscheidungen auf Landesebene zur Auftragsvergabe, Qualitätskontrolle und Aufgabenbeschreibung für private Dienstleister zu einer institutionellen Verankerung der privaten Sicherheit im Politikfeld geführt haben, die zuvor in dieser Form nicht gegeben war. Anders gewendet: Die gefällten Entscheidungen und die implementierten Maßnahmen haben Koordinationseffekte, Komplementaritäten, Lerneffekte und Effekte adaptiver Erwartungen generiert, die es wahrscheinlich erscheinen lassen, dass der nun betretene Policy-Pfad auch in den künftigen Jahren weiterverfolgt werden wird. ${ }^{16}$

Die Diskussion des Wandels und möglicher Mechanismen der Pfadabhängigkeit lässt darauf schließen, dass die Flüchtlingskrise und die politische Reaktion auf diese tatsächlich eine critical juncture für die Produktion von Innerer Sicherheit in der Bundesrepublik dargestellt hat. Auch wenn die unterschiedlichen von uns erhobenen Daten in der Flüchtlingskrise (mit besonderer Rücksicht auf die Problematik der Unterbringung) ein gemischtes und noch nicht abgeschlossenes Bild von potentiellem Policy-Wandel wie auch Gegentendenzen zeichnen, scheinen die freigelegten Veränderungen qualitativ doch von einer solchen Tiefe zu sein, dass sie eine nachhaltige Wirkung auf das gesamte Politikfeld der Inneren Sicherheit haben dürften - und zwar insbesondere mit Blick auf die zunehmend wichtigere Rolle privater Sicherheitsdienstleister. Dieses Ergebnis knüpft in dreierlei Hinsicht an die Literatur an: Zum ersten bezeugt es eine abnehmende Rolle des Staates als alleiniger Produzent von Sicherheit und die wachsende Bedeutung staatlicher Steuerungsfähigkeit im Sinne von "nodal governance“ (Johnston/Shearing 2003). Zum zweiten untermauert es die Diagnose eines doppelten Sicherheitsaufbaus in Deutschland, der weniger durch einen generellen Rückzug des Staates als durch eine verstärkte Kooperation geprägt ist (Beste 2009). Und zum dritten verweist es auf die Relevanz von Untersuchungen zur Entgrenzung von Sicherheit (Huysmans 20I4) und zur "Versicherheitlichung" von Politikfeldern bzw. Problemlagen (Buzan/Waever/De Wilde 1998).

\footnotetext{
I6 Daneben hat schließlich - ganz unabhängig von den genannten vier Sydow'schen Mechanismen - die Zunahme der Zahl der Polizistinnen und Polizisten pfadformierende Wirkung, da die Einstellungen in den vergangenen 24 Monaten über das Beamtenrecht quasi funktionalistisch eine langfristige Veränderung der Polizeistärken bei Bund und Ländern bedeuten.
} 
Inwieweit diese für den Fall Deutschland erkennbaren Tendenzen auch in anderen europäischen Ländern, die ähnlichen Situationen ausgesetzt waren, festgestellt werden können, bleibt eine offene Frage. Insbesondere aber für diejenigen Staaten mit hohen Zahlen ankommender Flüchtlinge, wie etwa die Republik Österreich aber auch Schweden, dürften vergleichbare Mechanismen zum Tragen kommen. Zumindest in Österreich deuten die in Folge der Flüchtlingskrise geführten Debatten über die Gewährleistung von Sicherheit und die Notwendigkeit, mehr Polizisten einzustellen (Parlament 2016), auf ähnliche Mechanismen hin, deren Auswirkungen bis hin zum sicherheitspolitischen Programm der aktuellen Regierung reichen (Zeit-Online 20I7).

\section{Literatur}

BAMF (20I7), Schlüsselzahlen Asyl, I. Halbjahr 20I7, Internet: http://www.bamf.de/SharedDocs/Anlagen/ DE/Publikationen/Flyer/flyer-schluesselzahlenasyl-halbjahr-20I7.pdf?__blob=publicationFile $(\mathrm{Zu}-$ griff: IO.OI.2018).

Buzan, Barry/ Ole Waever / Jaap De Wilde (1998), Security: A New Framework for Analysis, Boulder: Lynne Rienner.

$B D S W$ (2017), Sicherheitswirtschaft wächst um über 20 $\%$. Pressemitteilung des BDSW I9 / 2017, Internet: https://www.bdsw.de/presse/bdsw-pressemitteilungen/sicherheitswirtschaft-waechst-um-ueber-20 (Zugriff: 22.05.2017).

$B D S W / B D G W(2017)$, Sicherheitswirtschaft in Deutschland, Internet: https://www.bdsw.de/images/statistiksatz/Statistiksatz-BDSW-BDGW-20I7-OI24.pdf (Zugriff: 18.0I.2018).

Beste, Hubert (2009), Zur Privatisierung verloren geglaubter Sicherheit in der Kontrollgesellschaft, in Lange, Hans-Jürgen/Peter, H. Ohly/Jo Reicherts (Hg.), Auf der Suche nach neuer Sicherheit: Fakten, Theorien und Folgen, Wiesbaden: VS Verlag für Sozialwissenschaften, I83-202.

Beyer, Jürgen (2005), Pfadabhängigkeit ist nicht gleich Pfadabhängigkeit!: Wider den impliziten Konservatismus eines gängigen Konzepts, in: Zeitschrift für Soziologie, Vol. 34 (I), 5-2I.

$B K A$ (2016), Kriminalität im Kontext von Zuwanderung: Kernaussagen, Internet: https://www.bmi.bund.de/ SharedDocs/Downloads/DE/Kurzmeldungen/lageuebersicht-kriminalitaet-kontext-zuwanderung. pdf?__blob=publicationFile (Zugriff: 30.06.2016).

Boswell, Christina (2007), Migration Control in Europe After 9/II: Explaining the Absence of Securitization, in: Journal of Common Market Studies, Vol. 45 (3), 5896IO.
Bowden, Tom (1978), Beyond the Limits of Law. A Comparative Study of the Police in Crisis Politics, Harmondsworth: Penguin Books.

Bundesagentur für Arbeit (20I7), Beschäftigungsstatistik: Beschäftigte nach Wirtschaftszweigen - Deutschland, Länder, Internet: https://statistik.arbeitsagentur.de/nn_217696/Statischer-Content/Rubriken/ Beschaeftigung/Beschaeftigte/Beschaeftigte-nachWirtschaftsabteilungen-Wirtschaftsgruppen.html (Zugriff: 22.05.2017).

Bundesheer (2016), Flüchtlinge: Das Bundesheer hilft, Internet: http://www.bundesheer.at/archiv/a2015/ fluechtlingshilfe/index.shtml (Zugriff: 29.05.2018).

BMI (2017), Bundeshaushalt, Internet: https://www. bundeshaushalt-info.de/\#/2017/soll/ausgaben/einzelplan/o6.html (Zugriff: IO.OI.2018).

Bundesministerium für Inneres (2016), Asylstatistik 2015, Internet: http://www.bmi.gv.at/30I/Statistiken/files/ Jahresstatistiken/Asyl_Jahresstatistik_2015.pdf (Zugriff: 12.12.20I7).

Collier, Ruth Berins/David Collier (I99I), Shaping the political arena. Princeton: Princeton University Press.

Der Standard (2015), Bund wegen Flüchtlingen unter Druck, in: Der Standard, 16.12.2015, S.8.

Die Presse (2014), Asyllösung perfekt, aber Landeschefs stellen Bedingungen, Internet: https://diepresse. $\mathrm{com} /$ home/politik/innenpolitik/4598509/Asylloesung-perfekt-aber-Landeschefs-stellen-Bedingungen (Zugriff: 18.0I.2018).

Forst, Brian (1999), Policing with Legitimacy, Equity, and Efficiency, in: Forst, Brian/ Peter K. Manning (Hg.), The Privatization of Policing, Washington D.C.: Georgetown University Press, I-48.

Frevel, Bernhard/Michaela Wendekamm (Hg.) (2017), Sicherheitsproduktion zwischen Staat, Markt und Zivilgesellschaft, Wiesbaden: Springer VS.

Gimenez-Salinas, Andrea (2004), New approaches regarding private/public security, in: Policing and Society, Vol. I4 (2), I58-I74.

Glaeßner, Gert-Joachim (2010), A Change of Paradigm: Law and Order, Anti-Terrorism Policies, and Civil Liberties in Germany, in: German Politics, Vol. I9 (3-4), 479-496.

Hall, Peter/David Soskice (Hg.) (200I), Varieties of Capitalism, Oxford: Oxford University Press.

Hirschmann, Nathalie (2016), Sicherheit als professionelle Dienstleistung und Mythos. Eine soziologische Analyse der gewerblichen Sicherheit, Wiesbaden: Springer VS.

Huysmans, Jef (2014), Security Unbound. Enacting Democratic Limits, Abingdon: Routledge.

Huysmans, Jef (2000), The European Union and the Securitization of Migration, in: Journal of Common Market Studies, Vol. 38 (5), 75I-777. 
Jäckle, Sebastian/ Pascal D. König (20I7), The dark side of the German 'welcome culture': Investigating the causes behind attacks on refugees in 2015, in: West European Politics, Vol. 4O (2), 223-25I.

Johnston, Les/Clifford D. Shearing (2003), Governing Security: Explorations in Policing and Justice, Abingdon: Routledge.

Kirsch, Benno (2003), Private Sicherheitsdienste im öffentlichen Raum: Formen und Folgen der Zusammenarbeit mit der Polizei in Berlin und Frankfurt am Main, Wiesbaden: Westdeutscher Verlag.

Knill, Christoph/ Jale Tosun (2012), Public Policy: A New Introduction, London: Palgrave Macmillan.

Kötter, Matthias (2007), Pfade des Sicherheitsrechts: Begriffe von Sicherheit und Autonomie im Spiegel der sicherheitsrechtlichen Debatte der Bundesrepublik Deutschland, Baden-Baden: Nomos.

Löfstrand, Cecilia Hansen/ Bethan Loftus/ Ian Loader (2016), Doing 'dirty work': Stigma and esteem in the private security industry, in: European Journal of Criminology, Vol. I3 (3), 297-3I4.

McGregor, Douglas (1960), The human side of enterprise, New York: Mac Graw-Hill.

Mitteldeutscher Rundfunk (2015), Stahlknecht will Wachpolizisten per Erlass einstellen, Internet: http://www. mdr.de/sachsen-anhalt/stahlknecht-erla--wachpolizeiroo.html (Zugriff: 3I.IO.2016).

Parlament (2016), Parlamentskorrespondenz Nr. I240 vom I6.II.20I6: Budget für Innere Sicherheit wird 2017 um 440 Mio. Euro aufgestockt, Internet: https://www.parlament.gv.at/PAKT/PR/JAHR_2016/ PKI24O/ (Zugriff: 2I.I2.20I7).

Pierson, Paul (2004), Politics in time: History, institutions, and social analysis, Princeton: Princeton University Press.

Pitschas, Rainer (Hg.) (2002), Kriminalprävention und „Neues Polizeirecht": Zum Strukturwandel des Verwaltungsrechts in der Risikogesellschaft, Berlin: Duncker \& Humblot.

Prätorius, Rainer (Hg.) (2002), Wachsam und kooperativ?: Der lokale Staat als Sicherheitsproduzent, Baden-Baden: Nomos.

Schmidt, Manfred G. (1987), West Germany: The Policy of the Middle Way, in: Journal of Public Policy, Vol. 7 (I), I35-I77.

Smith, Martin J./Adam White (2014), The paradox of security regulation: Public protection versus normative legitimation, in: Policy \& Politics, Vol. 42 (3), 42 I-437.

Schuster, Armin (2015), Armin Schuster MdB: Eine Milliarde mehr für das BMI - Haushalt der Sicherheitsbehörden massiv gestärkt, Internet: http:// armin-schuster.eu/medien/pressemitteilungen/ armin-schuster-mdb-eine-milliarde-mehr-fur-dasbmi-haushalt-der-sicherheitsbehoerden-massivgestaerkt (Zugriff: 29.03.2016).
Shearing, Clifford D./ Philip C. Stenning (198I), Modern Private Security: Its Growth and Implications, in: Crime and Justice, Vol. 3, 193-245.

Staff, Helge/Georg Wenzelburger (2017), Innere Sicherheit und Justiz: Zwischen Großreform und Kontinuität, in: Hörisch, Felix/Stefan Wurster (Hg.), Das grünrote Experiment in Baden-Württemberg: Eine Bilanz der Landesregierung Kretschmann 20II-2016, Wiesbaden: Springer VS, 97-I23.

Sydow, Jörg/ Georg Schreyögg/ Jochen Koch (2009), Organizational Path Dependence: Opening the Black Box, in: Academy of Management Review, Vol. 34 (4), 689-709.

Tepe, Markus/ Pieter Vanhuysse (2013), Cops for hire?: The political economy of police employment in the German states, in: Journal of Public Policy, Vol. 33 (2), 165-199.

Van Steden, Ronald (2007), Privatizing Policing: Describing and Explaining the Growth of Private Security, Den Haag: Boom Juridische Uitgevers.

Van Steden/Ronald/Jaap De Waard (2013), ,Acting like Chameleons': On the McDonaldization of private security, in: Security Journal, Vol. 26 (3), 294-309.

Van Steden/ Ronald/ Rick Sarre (2007) The Growth of Privatized Policing: Some Cross-national Data and Comparisons, in: International Journal of Comparative and Applied Criminal Justice, Vol. 3I (I), 5I-7I.

Wakefield, Alison (2003), Selling Security: The Private Policing of Public Space, Cullompton: Willan Publishing.

Wenzelburger, Georg/Helge Staff (2016), German exceptionalism?: An empirical analysis of 20 years of law and order legislation, in: Politics \& Policy, Vol. 44 (2), 319-350.

Wenzelburger, Georg/Helge Staff (2018), Im Zweifel für mehr Sicherheit: Law-and-Order-Politik zwischen Terror und Flüchtlingskrise, in: Zohlnhöfer, Reimut/ Saalfeld, Thomas (Hg.), Zwischen Stillstand, Politikwandel und Krisenmanagement. Eine Bilanz der Regierung Merkel 2013-2017, Wiesbaden: Springer VS, 549-568.

White, Adam (2010), The Politics of Private Security, Basingstoke: Palgrave Macmillan.

Zeit-Online (2017), Rechte Hardliner in Schlüsselpositionen: Der künftige Kanzler überlässt der FPÖ die Minister für Inneres, Äußeres und Verteidigung. Der Bundespräsident fordert ihn zu einer europafreundlichen Politik auf, Internet: http://www.zeit.de/ politik/ausland/20I7-I2/oesterreich-kabinett-kurzbellen-fpoe-rechtspopulisten-hardliner (Zugriff: 2I.I2.2OI7). 


\section{Autoren}

Helge Staff ist wissenschaftlicher Mitarbeiter an der Professur für Policy-Analyse und Politische Ökonomie an der Technischen Universität Kaiserslautern, Deutschland. Neben seinen dissertationsbezogenen Forschungen zur Politischen Ökonomie der privaten Sicherheit beschäftigt er sich im Schwerpunkt mit Fragen der Policy-Analyse etwa zu Politiken der Inneren Sicherheit oder zur Weiterentwicklung von Policy-ProzessTheorien. Aktuelle Arbeiten wurden in Policy Studies (zu Parteieffekten durch Policy-Entrepreneure), im European Journal of Political Research (zur Politik der Inneren Sicherheit) und in Global Change, Peace \& Security (zur privaten Sicherheit) veröffentlicht.

Helge Staff, M.A.

Fachbereich Sozialwissenschaften, TU Kaiserslautern

Erwin-Schroedinger-Str.

67653 Kaiserslautern

Tel.: +49 (o)63I-205-4970

Georg Wenzelburger ist Professor für Policy-Analyse und Politische Ökonomie an der Technischen Universität Kaiserslautern, Deutschland. Seine Hauptforschungsinteressen liegen im Feld der vergleichenden PolicyForschung mit einem Schwerpunkt auf der Politik der Inneren Sicherheit, Wohlfahrtsstaatsreformen und der Finanzpolitik. Aktuelle Arbeiten wurden zum Beispiel im British Journal of Political Science (zu Wohlfahrtsstaatsreformen), im European Journal of Political Research (zur Politik der Inneren Sicherheit) und in Comparative European Politics (zu Austeritätspolitik) veröffentlicht.

Prof. Dr. Georg Wenzelburger

Fachbereich Sozialwissenschaften, TU Kaiserslautern

Erwin-Schroedinger-Str.

67653 Kaiserslautern

Tel.: +49 (o)63I-205-3840 